\title{
Association between preoperational blood profiles and post operational complications in patients suffering epithelial ovarian cancer
}

\begin{abstract}
Anemia, leukocytosis, thrombocytosis and other para neo plastic disorders have been seen in numerous malignancies especially in advanced stages. We studied the preoperational leukocytosis and thrombocytosis and clinical outcomes in patients with epithelial ovarian cancer. This study was carried out as a prospective cohort at the gynecology oncology department of Valiasr ward of Imam Hospital of Tehran university of Iran. The blood cells profile, post operational complications and overall survival have been investigated. The demographic characteristic and information concerning to specifications of ovarian cancer histopathologically, count of preoperational blood cells, post operational complications occurring after 30 days of surgery, recurrence and metastasize of disease during a year after surgery were investigated. Preoperational leukocytosis was associated with higher stage and grade and superficial and deep wound infections. Preoperational leukocytosis was associated with higher mortality rate and lower overall survival too. Further studies must pay attention to association between blood cells parameters and prediction of response to treatment option selected in epithelial ovarian cancer for choosing patients who are most profits of neo adjuvant chemotherapy.
\end{abstract}

Keywords: preoperational blood profiles, epithelial ovarian cancer, post operational complications
Volume 6 Issue 3 - 2017

\section{Setare Nasiri, Setare Akhavan, Zahra \\ Golshani, Mitra Modarres, Azamosadat Mousavi, Shahrzad Sheikh Hassani}

Gynecology Oncology of Imam Hospital, Tehran University of Medical Science, Iran

Correspondence: Setare Nasiri, Gynecologist oncologist of Tehran University, Imam Hospital of Tehran, Tehran, Iran, Tel 989|| 2556829, Fax 982 |6 | 92363 .

Email setare_n99@yahoo.com

Received: September 12,2016 | Published: November 17 2017
Abbreviations: CA 125, cancer antigen 125; GCSF, granulocyte cloning stimulating factor, CA 19-9,cancer antigen 199; HE4, human epididymis protein 4; IGF 2, insulin growth factor 2

\section{Introduction}

Leukocytosis is a para neo plastic syndrome which arises nearly in $30 \%$ of solid tumor. Anemia, leukocytosis, thrombocytosis and other para neo plastic disorders have been seen with numerous malignancies especially in advanced stages. ${ }^{1}$ Para neo plastic syndrome often is seen with gynecologic, lung, breast and hematologic malignancies. Recent studies on leukocytosis and other blood markers in disease's progression have shown the negative effect of leukocytosis on malignancies. ${ }^{2}$ Ovarian cancer is one of the main reasons of gynecologic cancer related death. All over the world about 238700 new cases and 151900 deaths in 2013 was diagnosed. ${ }^{3}$ Most cases are discovered in advanced stages. This problem is related to the nature of cancer and lack of sufficient screening program. Even when the patient with advanced disease who treated by cytoreductive surgery fallowed by adjuvant chemotherapy prognosis would be poor yet. So that 5years survival is just $50 \%{ }^{3}$ The known risk factors for unfavorable results are included: high age, high stage and grade, clear cell histology, excessive volume of ascites, the large volume of residual tumor in the first laparotomy. ${ }^{3}$ Recently molecular biomarkers have being increasingly studied in order to diagnosis and define of primary prognosis. The relationship between mononucleotide polymorphism in some of cancer related gens and survival of patients are also response to chemotherapy in ovarian cancer has been discovered. At this matter can be pointed out to mononucleotide poly morphism in IGF 2 can lead to exceed the recurrence and death. ${ }^{4}$ To study of the clinical outcomes of cancer stem cells and their superficial cell markers, there are some controversial about application of cell markers in ovarian cancer. ${ }^{5}$ Also many of the mentioned studies which have been carried out with micro assay methods lead to high pseudo positive results in addition these studies are very different that may be because of the difference in micro assay techniques or analysis methodology. ${ }^{6}$

In spite of the studies, analysis of leukocyte is a very simple method and there is a little technical difference among labs, more important than this fact, the studies about relationship between leukocyte and cancer prognosis show reasonable and similar results among different cancer. ${ }^{7}$ According to hematologic parameters thrombocytosis is known to be related to these factors included: higher CA125 level before surgery, higher volume of ascites and lower survival. Also previous studies showed anemia is related to lower progression free survival and overall survival in ovarian cancer. Inflammation has an important role in primary stages and its prognosis. So the value of systemic inflammatory response markers has been taken to consideration. ${ }^{8}$ Neutrophil to lymphocyte ratio is one of the most systemic inflammatory response markers which has been used to prediction of clinical result and prognosis in different cancers. However the information on efficacy in ovarian cancer is still limited. This disease is not a simple one and composed of heterogeneous tumors which have different morphological and molecular characteristic. The importance of pre operational leukocytosis, anemia and thrombocytosis, in post operational complications is not clear. The main goal of the present study is to evaluate the relationship between blood cells counts before surgery and post operational complications rate, within 30 days after surgery and survival of patients suffering from ovarian cancer. Previous studies in this area have been retrospective but our study is a retrospective cohort. 


\section{Materials and methods}

This study was carried out as a prospective cohort and done at the gynecology oncology department of Valiasr ward of Imam Hospital of Tehran university of Iran.

\section{Including criteria}

Patients with epithelial ovarian cancer have been referred to the gynecology oncology department.

\section{Excluding criteria}

Patients with the history of acute or chronic preoperational infection, neo adjuvant chemotherapy, simultaneously malignancy, corticosteroid therapy after surgery and GCSF infusion were excluded. The blood cells profile, post operational complications and overall survival were investigated. The demographic characteristic and information concerning to specifications of ovarian cancer histopathologically, count of preoperational blood cells, post operational complications occurring after 30days of surgery, recurrence and metastasize of disease during a year after surgery were investigated. Pre operational leukocytosis was described as the leukocyte count higher than 10000 in microliter and thrombocytosis as the platelet count higher than 400000 in microliter. For each patient fallowing information has been collected based on her medical documents such as age, parity, body mass index, stage and grade of disease and serum level of CA125.The complications during 30 days after surgery which were reregistered in patient's files have been also evaluated. These complications include as:
i. Myocardial infarction,
ii. Thromboembolism,
iii. Wound infection,
iv. Prolonged intubation,
v. Urinary tract infection,
vi. Wound infection and
vii. Need to blood transfusion.

Primary evaluation of all patients has been carried out by gynecologist oncologist and all of them were taken under cytoreductive surgery, followed by adjuvant chemotherapy as a single protocol. At the time of getting the results out of the research to show quantities variables we used frequency (\%). The statistical analysis of quantities and qualities variables with normal distribution has also been carried out by use of Chi Squared test and student's T test respectively. The Mann Whitery statistical test was used to evaluate quantities variables without normal distribution. Shapiro_wilk test was used to measurement of normal distribution. $\mathrm{P}$ value was considered $0 / 05$ in all the analysis. Cox regression test was considered for Hazarded ratio. ${ }^{9}$ According to use of information which had recorded before, it was possible to direct contact to the patient to obtain consent. To save patient privacy we allocate special cod for each person and in all of stages of recording and analysis we used cods. The most important limitation of our study was incompleteness of files in some cases. All patients filled the consent form and our proposal has been granted by the local ethics in our university.

\section{Results}

The mean age of the women enrolled in this study was 49/89+years old. The types of pathology of ovarian cancer are exhibited in Table 1. Mean serum level of CA 125 was $899 / 4+15$. There were $7,15,94$ and 8 persons in stages 1, 2, 3 and 4 previously. The mean absolute count of leukocyte and platelet were $11666+33$ and 359+100/7*10 6 previously. In review of post operation complications in 127patients, superficial infection $=7$, deep wound infection $=9$, thromboembolism event $=6$, myocardial infarction $=2$, prolonged intubation $=4$ and blood transfusion was done in 36 women. Patients had been fallowed for 23mounts. 23patients died and 47 women recurred within this period. The patients were divided into two groups; first, women who did not have leukocytosis before surgery and second who had leukocytosis before operation. According to Table 2, there was a significant correlation between preoperational leukocytosis with stage and grade and patients with preoperational leukocytosis are in higher stages and grads. (CI 95\%, 1/8-11/5 P value $=0 / 032 \mathrm{HR}=2 / 3)$ and $(\mathrm{CI} \% 95,1 / 01-$ $19 / 5 \mathrm{P}$ value $=0 / 015 \mathrm{HR}=1 / 6$ ) respectively. Also there was a correlation between post operation complications such as superficial and deep wound infections and preoperational leukocytosis. Women who have preoperational leukocytosis are higher superficial and deep infection: (CI 95\%, 2/9-21/8 P value=0/029 $\mathrm{HR}=6 / 4)$ and (CI \%95, 1/3-61 P value $=0 / 033 \mathrm{HR}=8 / 56$ ) respectively. Preoperational leukocytosis is associated with mortality rate significantly and death is higher in this group. $(\mathrm{HR}=26, \mathrm{P}$ value $=0 / 002)$. According to Table 2, preoperational thrombocytosis was associated with deep wound infection and patient with preoperational thrombocytosis, have higher rate of deep infection: (CI \%95, 1/8-54/7 P value $=0 / 020 \mathrm{OR}=15 / 04)$, also mortality rate significantly was higher in women with preoperational thrombocytosis (P value $=0 / 006)$.

Table I Patients characteristics based on leukocyte count:preoperational leukocytosis is associated with higher stage and grade and superficial and deep wound infections. Preoperational leukocytosis is associated with higher mortality rate and lower overall survival too

\begin{tabular}{|c|c|c|c|c|}
\hline Feature reviewed & & Leukocyte count $<10000$ & Leukocyte count $>10000$ & P value \\
\hline Number of Patients & & 62 & 65 & ----- \\
\hline Mean of Age & & $11.67 \pm 50.05$ & $11.55 \pm 49.74$ & 0.88 \\
\hline Mean of CA125 & & $1458.62 \pm 785.72$ & $1645.28 \pm 1066.14$ & 0.42 \\
\hline \multirow{4}{*}{ Stage } & 1 & $1(2 \%)$ & $6(9.5 \%)$ & \multirow{4}{*}{0.026} \\
\hline & 2 & $7(11.5 \%)$ & $8(12.5 \%)$ & \\
\hline & 3 & $49(81.7 \%)$ & $45(70 \%)$ & \\
\hline & 4 & $3(5 \%)$ & $5(8 \%)$ & \\
\hline
\end{tabular}




\begin{tabular}{|c|c|c|c|c|}
\hline Feature reviewed & & Leukocyte count $<10000$ & Leukocyte count $>10000$ & Pvalue \\
\hline \multirow{3}{*}{ Grade } & 1 & $2(3 \%)$ & $5(8 \%)$ & \multirow{3}{*}{0.015} \\
\hline & 2 & $9(14.5 \%)$ & $16(25 \%)$ & \\
\hline & 3 & $51(82.5 \%)$ & $43(67 \%)$ & \\
\hline \multirow{2}{*}{ Superficial Wound Infections } & Positive & $0(0 \%)$ & $7(11 \%)$ & \multirow{2}{*}{0.01} \\
\hline & Negative & $62(100 \%)$ & $58(89 \%)$ & \\
\hline \multirow{2}{*}{ Deep Wound Infections } & Positive & $1(1.5 \%)$ & $8(12.5 \%)$ & \multirow{2}{*}{0.03} \\
\hline & Negative & $61(98.5 \%)$ & $57(87.5 \%)$ & \\
\hline \multirow{2}{*}{$\mathrm{UTI}^{\star}$} & Positive & $2(3 \%)$ & $0(0 \%)$ & \multirow{2}{*}{0.023} \\
\hline & Negative & $60(97 \%)$ & $65(100 \%)$ & \\
\hline \multirow{2}{*}{ Thromboembolism } & Positive & $3(5 \%)$ & $3(5 \%)$ & \multirow{2}{*}{$>0.999$} \\
\hline & Negative & $59(95 \%)$ & $62(95 \%)$ & \\
\hline \multirow{2}{*}{ Myocardial Infarction } & Positive & $2(3.5 \%)$ & $0(0 \%)$ & \multirow{2}{*}{0.23} \\
\hline & Negative & $60(96.5 \%)$ & $65(100 \%)$ & \\
\hline \multirow{2}{*}{ Need to Blood Transfusion } & Positive & $22(35.5 \%)$ & $14(16 \%)$ & \multirow{2}{*}{0.08} \\
\hline & Negative & $40(66.5 \%)$ & $51(84 \%)$ & \\
\hline \multirow{2}{*}{ Prolonged Intubation } & Positive & $3(5 \%)$ & $1(1.5 \%)$ & \multirow{2}{*}{0.35} \\
\hline & Negative & $59(95 \%)$ & $64(98.5 \%)$ & \\
\hline \multirow{2}{*}{ Recurrence } & Positive & $21(34 \%)$ & $26(40 \%)$ & \multirow{2}{*}{0.5} \\
\hline & Negative & $41(66 \%)$ & $39(60 \%)$ & \\
\hline \multirow[b]{2}{*}{ Mortality } & Positive & $22(35.5 \%)$ & $1(1.5 \%)$ & \multirow{2}{*}{$0.001>$} \\
\hline & Negative & $40(64.5 \%)$ & $64(98.5 \%)$ & \\
\hline
\end{tabular}

\section{*Upper Urinary Tract Infection.}

Table 2 Patients characteristics based on platelet count: Preoperational thrombocytosis is associated with higher mortality rate and overall survival too

\begin{tabular}{|c|c|c|c|c|}
\hline Feature reviewed & & Platelet count $<\mathbf{4 0 0 0 0 0}$ & Platelet count $>400000$ & P value \\
\hline Patients Number & & 78 & 49 & \\
\hline Mean of Age & & $12.21 \pm 50.33$ & $10.54 \pm 49.18$ & 0.588 \\
\hline Mean of CA125 & & $1613 \pm 1021.64$ & $1455.21 \pm 707.37$ & 0.271 \\
\hline \multirow{4}{*}{ Stage } & 1 & $2(2.5 \%)$ & $5(10 \%)$ & \multirow{4}{*}{0.217} \\
\hline & 2 & $8(10.5 \%)$ & $7(14 \%)$ & \\
\hline & 3 & $60(80 \%)$ & $34(69 / \%)$ & \\
\hline & 4 & $5(6.5 \%)$ & $3(6 \%)$ & \\
\hline \multirow{3}{*}{ Grade } & 1 & $2(2.5 \%)$ & $5(10.5 \%)$ & \multirow{3}{*}{0.063} \\
\hline & 2 & $13(17 \%)$ & $12(25 \%)$ & \\
\hline & 3 & $63(80.5 \%)$ & $31(66.5 \%)$ & \\
\hline \multirow{2}{*}{ Superficial Wound Infection } & Positive & $2(2.5 \%)$ & $5(10 \%)$ & \multirow{2}{*}{0.107} \\
\hline & Negative & $76(97.5 \%)$ & $44(90 \%)$ & \\
\hline \multirow{2}{*}{ Deep Wound Infection } & Positive & $1(1 \%)$ & $8(16 \%)$ & \multirow{2}{*}{0.002} \\
\hline & Negative & $77(99 \%)$ & $41(84 \%)$ & \\
\hline \multirow{2}{*}{ UTI } & Positive & $0(0 \%)$ & $2(4 \%)$ & \multirow{2}{*}{0.07} \\
\hline & Negative & $78(100 \%)$ & $47(96 \%)$ & \\
\hline
\end{tabular}


Table Continued

\begin{tabular}{|c|c|c|c|c|}
\hline Feature reviewed & & Platelet count $<400000$ & Platelet count $>\mathbf{4 0 0 0 0 0}$ & P value \\
\hline \multirow{2}{*}{ Thromboembolism } & Positive & $6(8 \%)$ & $0(0 \%)$ & \multirow{2}{*}{0.081} \\
\hline & Negative & $72(92 \%)$ & $49(100 \%)$ & \\
\hline \multirow{2}{*}{ Myocardial Infarction } & Positive & $2(3 \%)$ & $0(0 \%)$ & \multirow{2}{*}{0.552} \\
\hline & Negative & $76(97 \%)$ & $49(100 \%)$ & \\
\hline \multirow{2}{*}{ Need to Blood Transfusion } & Positive & $28(36 \%)$ & $8(16 \%)$ & \multirow{2}{*}{0.017} \\
\hline & Negative & $50(64 \%)$ & $41(84 \%)$ & \\
\hline \multirow{2}{*}{ Prolonged Intubation } & Positive & $4(5 \%)$ & $0(0 \%)$ & \multirow{2}{*}{0.159} \\
\hline & Negative & $74(95 \%)$ & $49(100 \%)$ & \\
\hline \multirow{2}{*}{ Recurrence } & Positive & $29(37 \%)$ & $18(36 \%)$ & \multirow{2}{*}{0.5} \\
\hline & Negative & $49(63 \%)$ & $31(64 \%)$ & \\
\hline \multirow{2}{*}{ Mortality } & Positive & $21(27 \%)$ & $2(4 \%)$ & \multirow{2}{*}{0.01} \\
\hline & Negative & $57(73 \%)$ & $47(96 \%)$ & \\
\hline
\end{tabular}

\section{Discussion}

patients with ovarian cancer and 43 normal women were studied. There was a significant difference among two groups. The patients

Generally tumors can affect blood cells parameters. There are multiple ways known about them including hemorrhage into the tumor, invasion to bone marrow, pre inflammatory cytokines and free radicals. All of effects are named as paraneoplastic syndrome. Correlation between anemia and more progressive disease and worse prognosis is described in multiple cancers such as GI, breast, head and neck, and lung. Usually tumors are associated with the changes in blood cells count. In the research by Chen in 2015 in china was designed about association between pre operational anemia, leukocytosis and thrombocytosis, and survival in patient with ovarian cancer. 816 women had been enrolled in this study, and there were anemia, leukocytosis, thrombocytosis, $13 / 4 \%, 16 / 7 \%$ and $22 / 8 \%$, respectively. Also preoperational anemia, leukocytosis, thrombocytosis were related with these factors including poor differentiated tumor cells, higher stages, lymph node involvement, residual disease after surgery larger than $1 \mathrm{~cm}$, ascites volume higher than $1000 \mathrm{cc}$, serum level of CA 125 higher than 675, and more recurrence of cancer. With Cox proportional hazarded model analysis has been proved that the thrombocytosis was an in depended factor for progression free survival and overall survival, and leukocytosis has closed relation with more malignant behavior and poorer prognosis.

Ashraf in her study in 2016 in Iran has discussed the association between neutrophil to lymphocyte ratio and platelet to lymphocyte ratio and operational outcomes $\mathrm{n}$ ovarian cancer. In this study 200 women were enrolled between 20052015 as a retrospective review. There was a significant relationship between surgical out come and both of ratios (Pvalue+0/011) the specificity and sensitivity of neutrophil to lymphocyte ratio were $074 \%$ and $67 \%$, respectively additionally the specificity and sensitivity of platelet to lymphocyte ratio were $74 \%$ and $85 \%$, respectively. Finally they concluded that both of ratios are useful in predicting operational out come in patient with ovarian cancer and increasing of each of them leads to poor operational outcome. ${ }^{10}$ Another study concluded that higher neutrophil to lymphocyte ratio and platelet to lymphocyte ratio and higher CA 125 serum level can be used for differentiation between malignant and benign mass. ${ }^{11}$ In Xing's study in 2016 in China monocyte to lymphocyte ratio in ovarian cancer was evaluated.133 whose monocyte to lymphocyte ratio was higher than $0 / 23$ in compare with her which the ratio is lesser than $0 / 23$ had higher serum levels of HE4, CA19-9 and CA125. There was significant difference in overall survival in two groups too. ${ }^{12}$ In the study carried out by Barbo in 2015 in United States the role of leukocytosis and thrombocytosis has been reviewed on post operational complications and mortality rate. Primary surgery was done and major complications included: myocardial infarction, thromboembolism, deep wound infection and prolonged intubation.

Minor complications included: urinary tract infections, superficial wound infection and need to blood transfusion. Out of 1075 patients who participated in this study, $9 / 6 \%$ had thrombocytosis and $17 / 9 \%$ had leukocytosis and $4 / 9 \%$ of patients have both of them synchronously. In mono variant analysis both of leukocytosis and thrombocytosis were related to the major complications but there was no evidence of the role of those factors and mortality rate. But the risk of major complications and mortality rate as well among the patients with both factors could be increased. After logistic regression analysis with adjust by age and co morbidity has been seen that the major complications however were related to preoperational thrombocytosis and leukocytosis. Finally researchers concluded that these factors were associated with 2 fold increase of post operational complications. ${ }^{13}$ In the retrospective study by so in 2014 in south chorea, pre operational leukocytosis was evaluated in order to predict overall survival of epithelial ovarian cancer. She was under taken debunking and adjuvant platinum base chemotherapy. Multi variant analysis showed preoperational leukocytosis was an independent prognostic factor to reduce overall survival. Finally they agreed it is necessary to get proved with the prospective trials. ${ }^{14}$ Stabuszewska in their study in 2015 in Poland have analyzed the role of platelets count as the prognostic factor in patient with ovarian cancer. There is a direct association between pre operational thrombocytosis with fallowing factors included: high grade, high stage and overall survival. So it was seemed platelet count could be an effective prognostic preoperational factor. $^{15}$ The study by Gunderson in 2013 in USA showed that preoperational leukocytosis despite of thrombocytosis has nothing to do with patient's prognosis. ${ }^{16}$ 
The role of preoperational thrombocytosis and thrombocytopenia after 6 courses of adjuvant chemotherapy on survival of patients with advanced ovarian epithelial cancer was studied by Lee in 2011 in South chorea. The final conclusion was that only thrombocytosis was associated with poor prognosis and low overall survival. ${ }^{17}$ In another study by Kim in 2011 in South chorea the impact of duration of anemia while chemotherapy was done in patient with epithelial ovarian cancer was investigated. This study was shown that if hemoglobin lesser than $10 \mathrm{mg} / \mathrm{dl}$ lasted more than 20\%of chemotherapy duration was associated with lower 5years survival. ${ }^{18}$ Causes of anemia in cancers are different included: iron metabolism disorders, extra corpuscular hemolysis and cell catabolism due to tumor invasion, and paracrine signaling factors such as interleukin-1, tumor necrosis factor. ${ }^{19}$ Recently it has been proven that higher expression of erythropoietin is associated with lymph node involvement and worse prognosis in endometrial cancer. Our knowledge about correlation between white blood cells count before surgery and prognosis of malignancy is incomplete. In one study about gastric cancer it was shown that preoperational absolute granulocyte count less than $6000 \mathrm{in} \mathrm{mm}^{3}$,

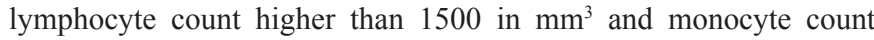
between 3000-9000 are the independent factors for poor prognosis and other studies were shown leukocytosis is associated with poor prognosis in lung cancer, colon cancer, endometrial cancer and cervical cancer. ${ }^{20-24}$

Similar result is seen in Chens study. ${ }^{9}$ In Chen study the result was, patients with epithelial ovarian cancer with higher malignant tumor characteristics, have more leukocytosis and lower overall survival. The main cause was systemic inflammation due to malignant cells activation which leads to DNA damage, apoptosis inhibition and increase of angiogenesis around tumor, finally cause to growth, spread and Metastasize. ${ }^{9}$ Previous studies was shown that thrombocytosis is associated with characteristics of patients and poor prognosis in gastric cancer. In Unals study the platelets to lymphocyte ratio has been introduced as predicting factor for response rate and prognosis in non-small cell carcinoma of lung. ${ }^{25}$ Role of thrombocytosis in ovarian cancer was shown by Stone too. ${ }^{25}$ Another study established that thrombocytosis is associated with pulmonary thromboembolism and tumor cell proliferation. ${ }^{26}$ Platelets can produce multiple growth factors such as thrombospondin and vascular endothelial growth factor. ${ }^{26}$

All of them are mitogen factors. The mitogen factors can proliferate and facilitate cell to cell adhesion leads to growth and metastasize. ${ }^{27}$ Another significant point is that interleukin-6 and granulocyte macrophage cloning stimulating factor cause trombopoesies and we know ovarian epithelial cancer secrets high level of interleukin-6.According to Barbers study either leukocytosis and thrombocytosis are associated with higher risk of serious post operational complications such as myocardial infarction, pneumonia, deep vein thrombosis, second surgery for hemorrhagic events, renal insufficiency and prolonged intubation independently and if patient has both of them (thrombocytosis and leukocytosis, mortality rate can be 4 times higher. ${ }^{28,13}$ The first treatment for epithelial ovarian cancer may be surgery or neo adjuvant chemotherapy. If the cancer is known to be respectable in the preoperational evaluation or there is multiple co morbidity or low performance status of her, first choice of treatments should be neo adjuvant chemotherapy. In previous studies about comparing of neo adjuvant and primary surgery in epithelial ovarian cancer were shown lower rate of post operational complications grade 3, 4 in chemotherapy rather than surgery. ${ }^{29,30}$
So for high risk patients, more dilution must be considered to select treatment options. Preoperational leukocytosis and thrombocytosis are 2 factors in keeping another factor such as age and general condition can detect patients with higher post operational complications. In addition detection of leukocyte and platelet counts are identifiable and interpretable easily and can be useful for select treatment option. As a result we conclude preoperational leukocytosis is associated with higher stage and grade and superficial and deep wound infections. Preoperational leukocytosis is associated with higher mortality rate and lower overall survival too. Preoperational thrombocytosis is associated with higher mortality rate and overall survival too.

\section{Conclusion}

The influence of malignant cells on hematopoiesis and inflammation, not only has molecular aspect but also has many clinical effects, including how to respond to treatment. Further studies must pay attention to association between blood cells parameters and prediction of response to treatment option selected in epithelial ovarian cancer for choosing patients who are most profits of neo adjuvant chemotherapy.

\section{Acknowledgements}

Setare Nasiri drafted the manuscript and reported the initial observation of the patient; Setare Nasiri and Setare Akhavan coordinated the national review and helped to draft the manuscript; and helped to retrieve the patient's data from the national database and coordinated the pathological review; Golshan and Mousavii performed the histological analysis of the specimen and animated the pathological discussion $\backslash$ Sheikh Hassani and Modarres managed the patient and helped to draft the manuscript. All authors are in reproductive health research center, Tehran University of medical sciences Tehran Iran and read and approved the final manuscript.

\section{Conflict of interest}

The authors do not report any financial or personal connections with other persons or organizations, which might negatively affect the content of this publication and/or claim authorship rights to this publication.

\section{References}

1. Pandit S, Choudhury S, Das SK, et al. Leucocytosis in a case of lung cancer: infection or paraneoplastic syndrome?-dilemma in diagnosis and treatment. Med J Malaysia. 2011;67(5):542-543.

2. Mabuchi S, Matsumoto Y, Isohashi F, et al. Pretreatment leukocytosis is an indicator of poor prognosis in patients with cervical cancer. Gynecol Oncol. 2011;122(1):25-32.

3. Raungkaewmanee S, Tangiitgamol S, Manusirivithaya S, et al. Platelet to lymphocyte ratio as a prognostic factor for epithelial ovarian cancer. $J$ Gynecol Oncol. 2012;23(4):265-273.

4. Meng QH, Xu E, Hildebrandt MA, et al. Genetic variants in the fibroblast growth factor pathway as potential markers of ovarian cancer risk, therapeutic response, and clinical outcome. Clin Chem. 2014;60(1):222232.

5. Kuroda T, Hirohashi Y, Torigoe T, et al. ALDH1-high ovarian cancer stemlike cells can be isolated from serous and clear cell adenocarcinoma cells, and ALDH1 high expression is associated with poor prognosis. PloS one. 2013;8(6):e65158. 
6. Yang N, Kaur S, Volinia S, et al. MicroRNA microarray identifies Let-7 as a novel biomarker and therapeutic target in human epithelial ovarian cancer. Cancer Res. 2008;68(24):10307-10314.

7. Tomita M, Shimizu T, Hara M, et al. Preoperative leukocytosis, anemia and thrombocytosis are associated with poor survival in non-small cell lung cancer. Anticancer Res. 2009;29(7):2687-2690.

8. Hanahan D, Weinberg RA. Hallmarks of cancer: the next generation. Cell. 2011;144(5):646-674

9. Chen Y, Zhang L, Liu WX, et al. Prognostic significance of preoperative anemia, leukocytosis and thrombocytosis in chinese women with epithelia ovarian cancer. Asian Pac J Cancer Prev. 2015;16(3):933-939.

10. Ashrafganjoei T, Mohamadianamiri M, Farzaneh F, et al. Investigating preoperative hematologic markers for prediction of ovarian cancer surgical outcome. Asian Pac J Cancer Prev. 2016;17(3):1445-1448.

11. Yildirim MA, Seckin KD, Togrul C, et al. Roles of neutrophil/lymphocyte and platelet/lymphocyte ratios in the early diagnosis of malignant ovarian masses. Asian Pac J Cancer Prev. 2014;15(16):6881-6885.

12. Xiang J, Zhou L, Li X, et al. Preoperative monocyte-to-lymphocyte ratio in peripheral blood predicts stages, metastasis, and histological grades in patients with ovarian cancer. Transl Oncol. 2017;10(1):33-39.

13. Barber EL, Boggess JF, Van Le L, et al. Association of preoperative thrombocytosis and leukocytosis with postoperative morbidity and mortality among patients with ovarian cancer. Obstet Gynecol. 2015;126(6):1191-1197.

14. So KA, Hong JH, Jin HM, et al. The prognostic significance of preoperative leukocytosis in epithelial ovarian carcinoma: A retrospective cohort study. Gynecol Oncol. 2014;132(3):551-555.

15. Słabuszewska Jóźwiak A, Dmoch Gajzlerska E, et al. The prognostic significance of thrombocytosis in ovarian cancer. Ann Agric Environ Med. 2014;22(4):731-735.

16. Gunderson CC, Slaughter KN, Thomas ED, et al. Is leukocytosis harbinger of poor prognosis in ovarian cancer akin to thrombocytosis? Gynecologic Oncology. 2013;131(1):263.

17. Weiss G, Goodnough LT. Anemia of chronic disease. $N$ Engl J Med. 2005;352(10):1011-1023.

18. Qiu MZ, Xu RH, Ruan DY, et al. Incidence of anemia, leukocytosis, and thrombocytosis in patients with solid tumors in China. Tumour Biol. 2010;31(6):633-641.
19. Seo KH, Lee HS, Jung B, et al. Estrogen enhances angiogenesis through a pathway involving platelet-activating factor-mediated nuclear factor- $\kappa \mathrm{B}$ activation. Cancer Res. 2004;64(18):6482-6488.

20. Jung MR, Park YK, Jeong O, et al. Elevated preoperative neutrophil to lymphocyte ratio predicts poor survival following resection in late stage gastric cancer. J Surg Oncol. 2011;104(5):504-510.

21. Holgersson G, Sandelin M, Hoye E, et al. Swedish lung cancer radiation study group: the prognostic value of anaemia, thrombocytosis and leukocytosis at time of diagnosis in patients with non-small cell lung cancer. Med Oncol. 2012;29(5):3176-3182.

22. Mroczko B, Groblewska M, Wereszczyńska-Siemiatkowska U, et al Serum macrophage-colony stimulating factor levels in colorectal cancer patients correlate with lymph node metastasis and poor prognosis. Clin Chim Acta. 2007;380(1):208-212.

23. Njølstad TS, Engerud H, Werner HM, et al. Preoperative anemia, leukocytosis and thrombocytosis identify aggressive endometrial carcinomas. Gynecol Oncol. 2013;131(2):410-415.

24. Grivennikov SI, Greten FR, Karin M. Immunity, inflammation, and cancer. Cell. 2010;140(6):883-899.

25. Unal D, Eroglu C, Kurtul N, et al. Are neutrophil/lymphocyte and platelet/ lymphocyte rates in patients with non-small cell lung cancer associated with treatment response and prognosis? Asian Pac $J$ Cancer Prev. 2013;14(9):5237-5242.

26. Stone RL, Nick AM, McNeish IA, et al. Paraneoplastic thrombocytosis in ovarian cancer. N Engl J Med. 2012;366(7):610-618.

27. Khorana AA. Cancer and thrombosis: implications of published guidelines for clinical practice. Ann Oncol. 2009;20(10):1619-1630.

28. van Rossum AP, Vlasveld LT, Vlasveld IN, et al. Granulocytosis and thrombocytosis in renal cell carcinoma: a pro-inflammatory cytokine response originating in the tumour. Neth J Med. 2009;67(5):191-194.

29. Vergote I, Tropé CG, Amant F, et al. Neoadjuvant chemotherapy or primary surgery in stage IIIC or IV ovarian cancer. $N$ Engl $\mathrm{J} \mathrm{Med}$. 2010;363(10):943-953.

30. Kehoe S, Hook J, Nankivell M, et al. Primary chemotherapy versus primary surgery for newly diagnosed advanced ovarian cancer (CHORUS): an open-label, randomised, controlled non-inferiority trial. The Lancet. 2015;386(9990):249-257. 